This new feature will emphasize innovative and better ways to perform dermatologic surgery procedures. This article should be based on some evidence-based literature, but may describe the author's experience with a particular procedure without being a typical clinical research article. The Editor will consider ideas for topics. Any author who is considering writing an article should submit the title to Ronald L. Moy, MD, Editor-in-Chief, 100 UCLA Medical Plaza, Suite 590, Los Angeles, CA 90024.

\title{
Concepts of Sliding and Lifting Tissue Movement in Flap Reconstruction
}

\author{
Timothy M. Johnson, MD, ${ }^{* \dagger \ddagger ~ N e i l ~ S w a n s o n, ~ M D, ~} \$$ and Shan R. Baker, MD ${ }^{\dagger}$ \\ Departments of *Dermatology, ${ }^{\dagger}$ Otorhinolaryngology, and $\ddagger$ Surgery, University of Michigan Medical Center, \\ Ann Arbor, Michigan, and $\$$ Department of Dermatology and Otolaryngology, Oregon Health Sciences, \\ Portland Oregon
}

BACKGROUND. The optimal design of a skin flap requires an understanding of the concepts of tissue movement.

objective. The purpose of this manuscript was to demonstrate concepts of sliding and lifting tissue movement for flap reconstruction.

METHODS. Six similar defects located in the forehead-templeeyebrow region were repaired using a different skin flap.

RESUlTS. The specific flap design for a given defect is based on the answer to three predictable events that result from tissue transfer: Where is the tension? Where are the final incision lines? Where is the redundant tissue?

CONClusion. A mental exercise assessing all available reconstruction options should be performed for each individual patient and defect. Both patient and defect considerations need to be assessed. A thorough understanding of both anatomy and tissue movement is necessary for optimal skin flap reconstruction.
SEVERAL PREVIOUSLY PUBLISHED review articles and textbooks have eloquently and thoroughly explained the important concepts necessary for optimal functional and aesthetic soft tissue reconstruction of the face utilizing skin flaps. ${ }^{1-4}$ This article is intended to build on and bridge these previous publications with practical current concepts of tissue movement.

The desire of patients to undergo total nasal reconstruction with pedicle flaps and the compelling drive of surgeons to reconstruct these defects historically date long before the pre-Christian era. Relatively recent advances in anesthesia, understanding of anatomy, instrumentation, antibiotics, wound healing, and the creative minds of reconstructive surgeons have given physicians the ability to achieve results with flap reconstruction that would have been considered miraculous only 30-40 years ago.

Political and philosophical differences among surgical specialists in dermatology, otorhinolaryngology, plastic surgery, and ophthalmology still exist. However, interchange of knowledge and experience be-

T.M. Johnson, MD, N. Swanson, MD, and S.R. Baker, MD, have indicated no significant interest with commercial supporters.

Address correspondence and reprint requests to: Timothy M. Johnson, MD, University of Michigan/Dermatology, 1910 Taubman Center, Box 0314, Ann Arbor, MI 48109-0314. tween these specialties over the past two decades has resulted in a hybrid of surgical approaches and a better understanding of the repair of cutaneous defects. Most important, the people who gain the most from cooperative interchange between specialties are our patients.

A thorough understanding of both anatomy and tissue movement is necessary for optimal soft tissue reconstruction. ${ }^{1-5}$ A mental exercise assessing all available reconstructive options should be performed for each individual patient and defect. Defect considerations include the availability of adjacent and/or distant loose tissue, and the need for structure, function, cosmesis, and tumor surveillance. Patient considerations include cosmetic consciousness and desire, and general medical state. The simplest closure often provides the best results. However, when simpler techniques result in prolonged healing (granulation), excessive wound closure tension, distortion of surrounding free margins and structures, or inferior cosmesis, local flap or graft reconstruction should be considered.

\section{Tissue Movement Terminology}

A flap is defined as movement of tissue from one location to another with an intact vascular supply. Movement of 

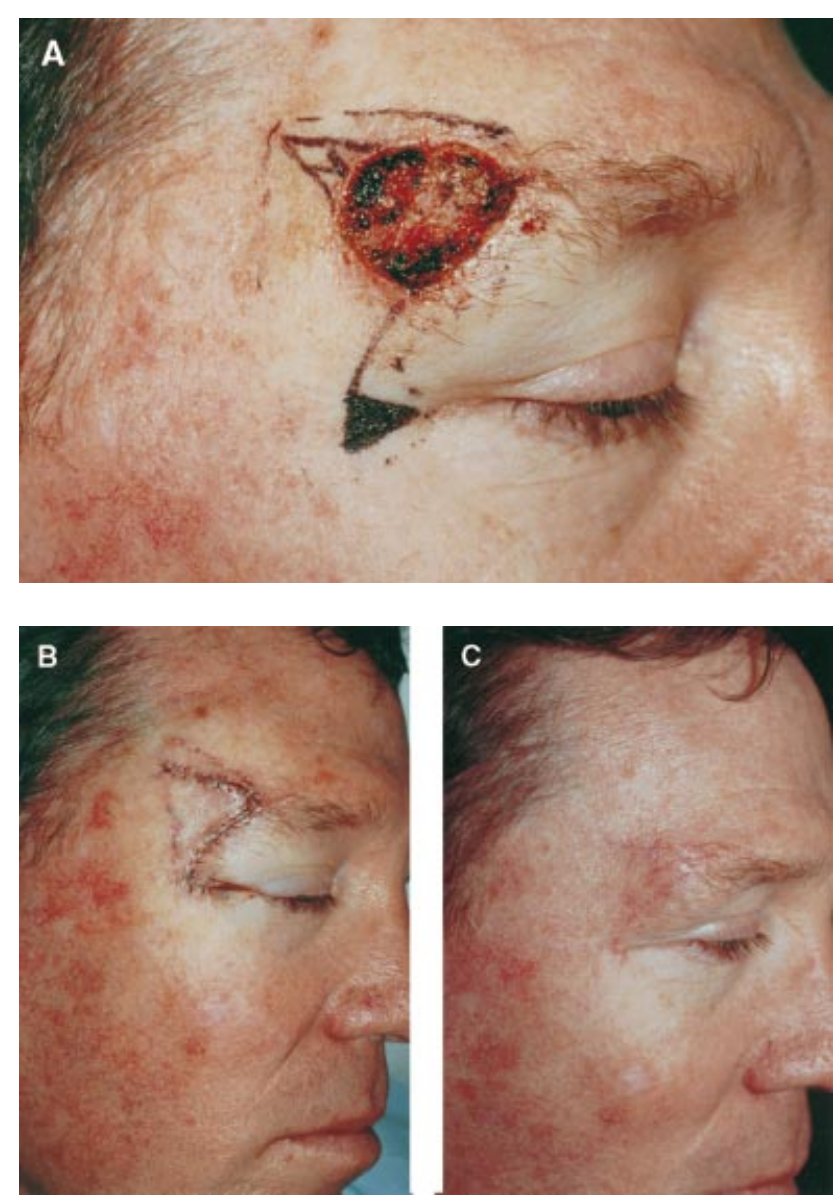

Figure 1. Patient 1. A 51-year-old man had a $2.0 \mathrm{~cm} \times 1.7 \mathrm{~cm}$ defect at the temple-forehead-eyebrow junction extending to the temporoparietal fascia following Mohs excision of a basal cell carcinoma (BCC). Loose tissue was identified at the temple. A) A Burrow's sliding flap was designed to take advantage of the loose adjacent tissue. The principle tension vector results from closure of the primary defect side to side, with no distortion of the free margin at the eyebrow. B) Scars lie in cosmetic unit boundary lines and a sleep line, and redundant skin is removed in an eyelid crease and within a sleep line. Care was taken to avoid damage to the superficial temporal branch of the facial nerve. C) Follow-up at 1 month.

local skin flaps historically have been categorized into three basic types: advancement, rotation, and transposition. ${ }^{1,2}$ Advancement flaps result in movement of tissue directly forward into a defect. Rotation flaps result in movement of a semicircular flap of tissue that rotates around a pivot point into a defect. Transposition flaps result in movement of a flap that is lifted and transposed, usually over an intervening bridge of normal skin, into a defect. Local skin flaps may have more than one mode of tissue movement.

Figure 2. Patient 2. A 28-year-old man had a $3.2 \mathrm{~cm} \times 3.5 \mathrm{~cm}$ defect at the lateral forehead adjacent to the temple and eyebrow following Mohs excision of a BCC. In contrast to patient 1, loose
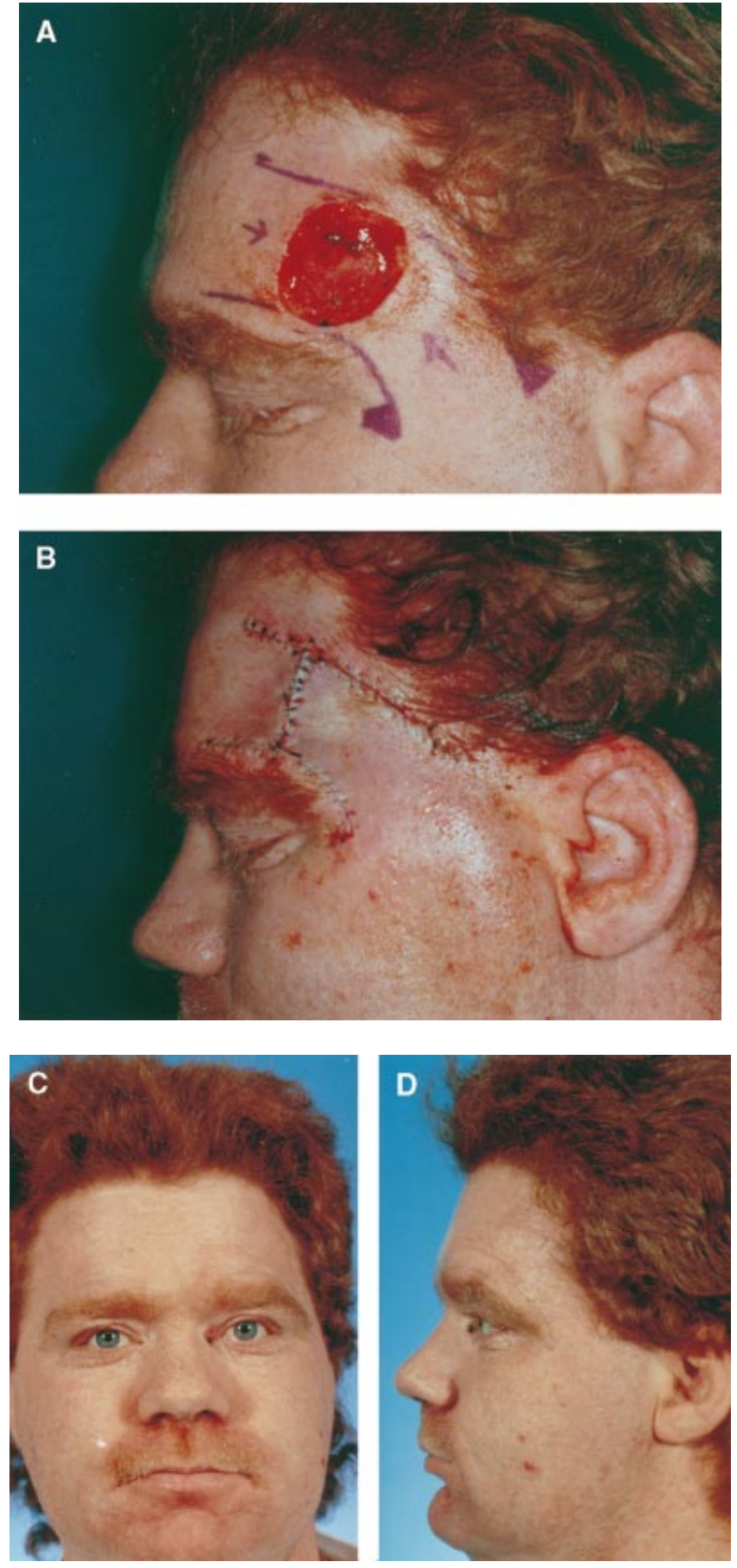

tissue was identified in both the temple and forehead areas. A) Bilateral sliding flaps with both advancement and some rotation (lateral flap) were designed to take advantage of the loose adjacent tissue reservoirs. The primary tension vector occurs side to side within the primary defect, with no distortion of the free margin at the eyebrow. B) Scars are located within natural forehead creases and redundant cones of tissue were excised in an eyelid crease and within the hairline. Care was taken to avoid damage to the temporal branch of the facial nerve located beneath the temporoparietal fascia laterally and frontalis muscle medially. In addition, care must be taken not to injure the supraorbital nerve by elevating the medially based flap in the subcutaneous plane. $C, D$ ) Follow-up at 3 months. 

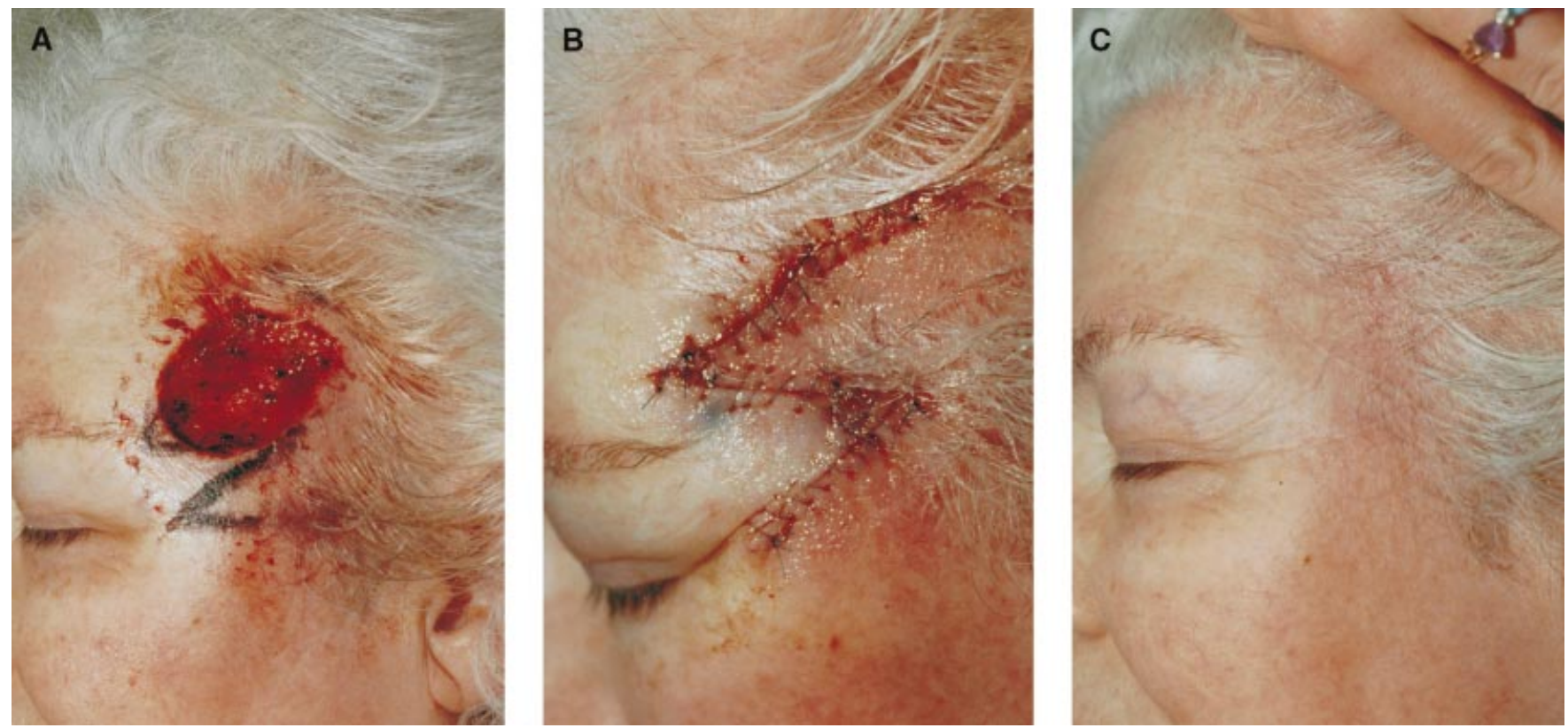

Figure 3. Patient 3. A 64-year-old woman had a $4.5 \mathrm{~cm} \times 3.4 \mathrm{~cm}$ defect at the temple-forehead-eyebrow junction extending to the temporoparietal fascia following Mohs excision of a BCC. Loose adjacent tissue was identified in the temple-cheek region. Half the defect involved the low-set hairline. The Burrow's sliding flap shown in patient 1 would result in a final incision line perpendicular to the relaxed free skin tension line at the temple-cheek area. A) A $30^{\circ}$ Webster lifting flap was designed to take advantage of the loose adjacent tissue, re-create the natural hairline, and place scars in the proper locations for optimal cosmetic results. B) Tension vectors result from closure of the secondary defect, resulting in no distortion at the free margin at the eyelid and eyebrow. The temporal branch of the facial nerve and temporal artery are located in this region. C) Follow-up at 6 months.

A more useful classification of flaps may be defined based on two instead of three types of tissue movement: sliding and lifting. ${ }^{6}$ Sliding flaps involve a sliding movement of tissue into the primary defect with a resulting secondary defect that consists principally of wounds of unequal length. The secondary defect is often repaired by the use of excising triangles of excess tissue along the long $\operatorname{arc}(\mathrm{s})$ or $\operatorname{limb}(\mathrm{s})$, or performing a back cut. Key sutures with maximal tension are used to close the primary defect. Advancement, rotation, and combinations of advancement/rotation flaps move tissue in a sliding fashion.

The second type of basic tissue movement involves lifting of flap tissue across or over a bridge of normal tissue to cover the primary defect. Key sutures with maximal tensions are used to close the secondary de-

Figure 4. Patient 4. A 54-year-old man had a $3.0 \mathrm{~cm} \times 2.1 \mathrm{~cm}$ defect at the forehead-temple-eyebrow area extending to the underlying muscle following Mohs excision of a BCC. Loose adjacent tissue was identified principally at the temple. A) A rhombic lifting flap was designed to take advantage of the loose adjacent tissue. The principle tension vector results from closure of the secondary defect, with no distortion of the free margin at the eyebrow and eyelid. B) Scars are located within natural lines and redundant skin was excised in an eyelid cease. The temporal branch of the facial nerve and temporal artery are located within this area. C) Followup at 4 months.
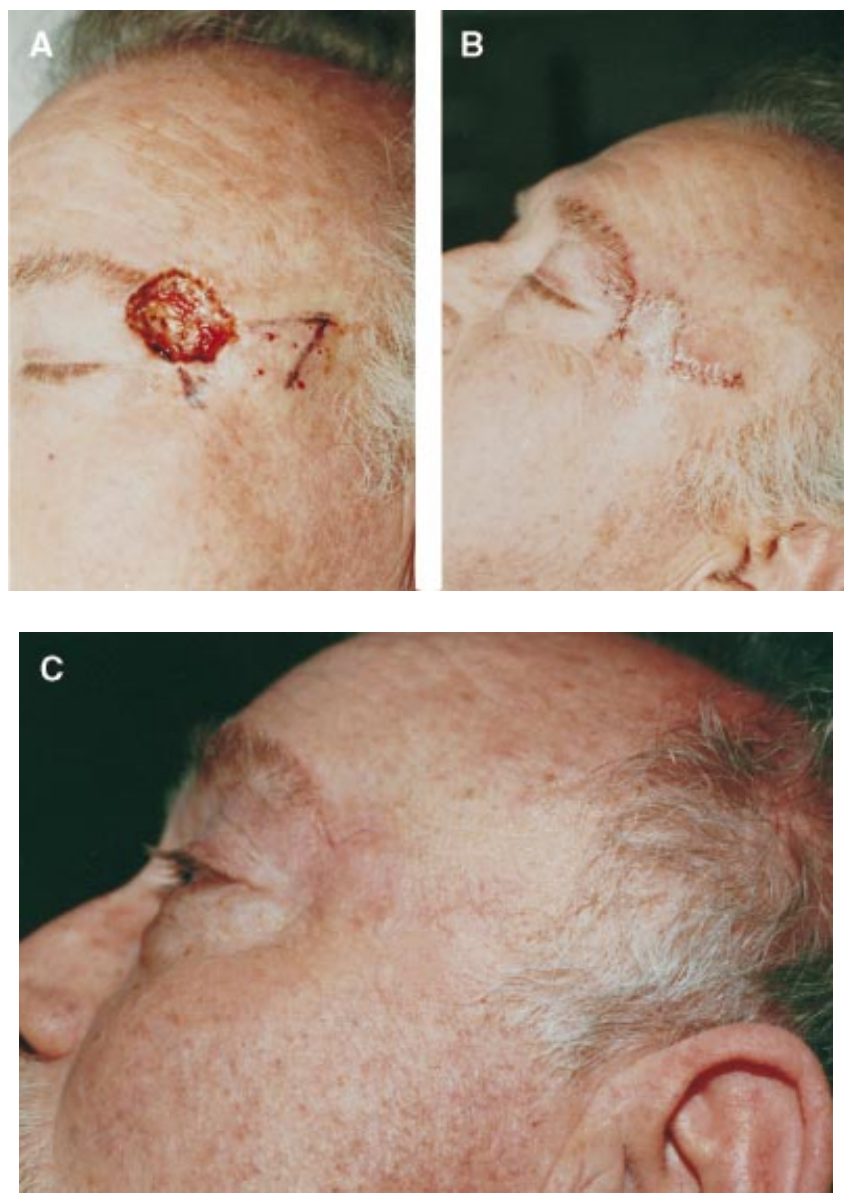

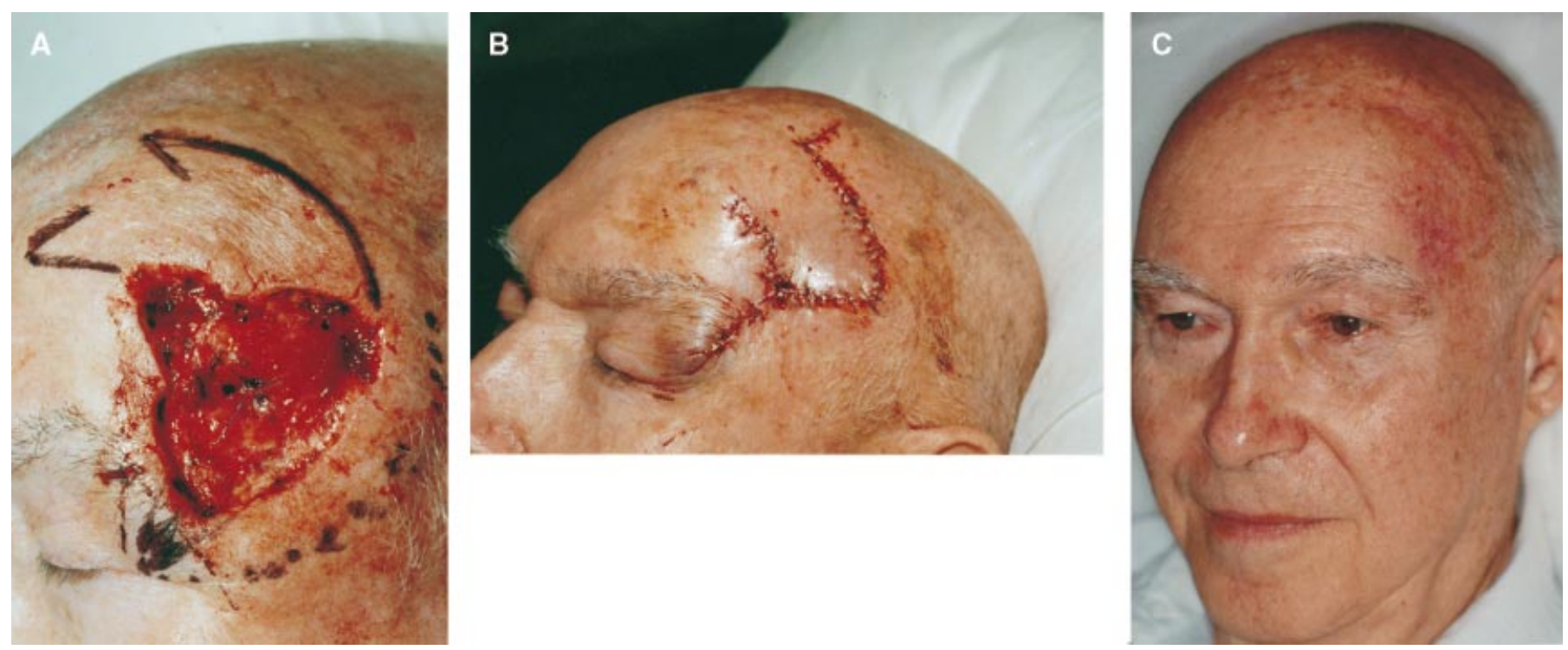

Figure 5. Patient 5. A 78-year-old man had a $6.0 \mathrm{~cm} \times 5.0 \mathrm{~cm}$ defect at the lateral forehead-eyebrow-temple junction extending to the temporoparietal fascia following Mohs excision of a BCC. Loose adjacent tissue was identified on the forehead, scalp, and temple. The inferior portion of the defect was repaired by sliding the eyebrow laterally secured with periosteal suspension suture. The remaining triangular defect was repaired using a sliding flap. Final tension vectors result in no distortion of the free margin at the eyelid and eyebrow. Secondary movement of scalp tissue was utilized to close the secondary defect. B) Scars are optimally located and redundant cones of tissue were removed using M-plasties at the eyelid and forehead (not the rhombic inked design) and a cone of tissue at the scalp. The temporal branch of the facial nerve, supraorbital nerve, and temporal artery are located within this area. C) Follow-up at 1 month. Figures $5 \mathrm{~A}-5 \mathrm{C}$ are reprinted with permission from Johnson TR, Nelson BR. Aesthetic reconstruction of skin cancer defects using flaps and grafts. Am J Cosmetic Surg 1992;259,260.
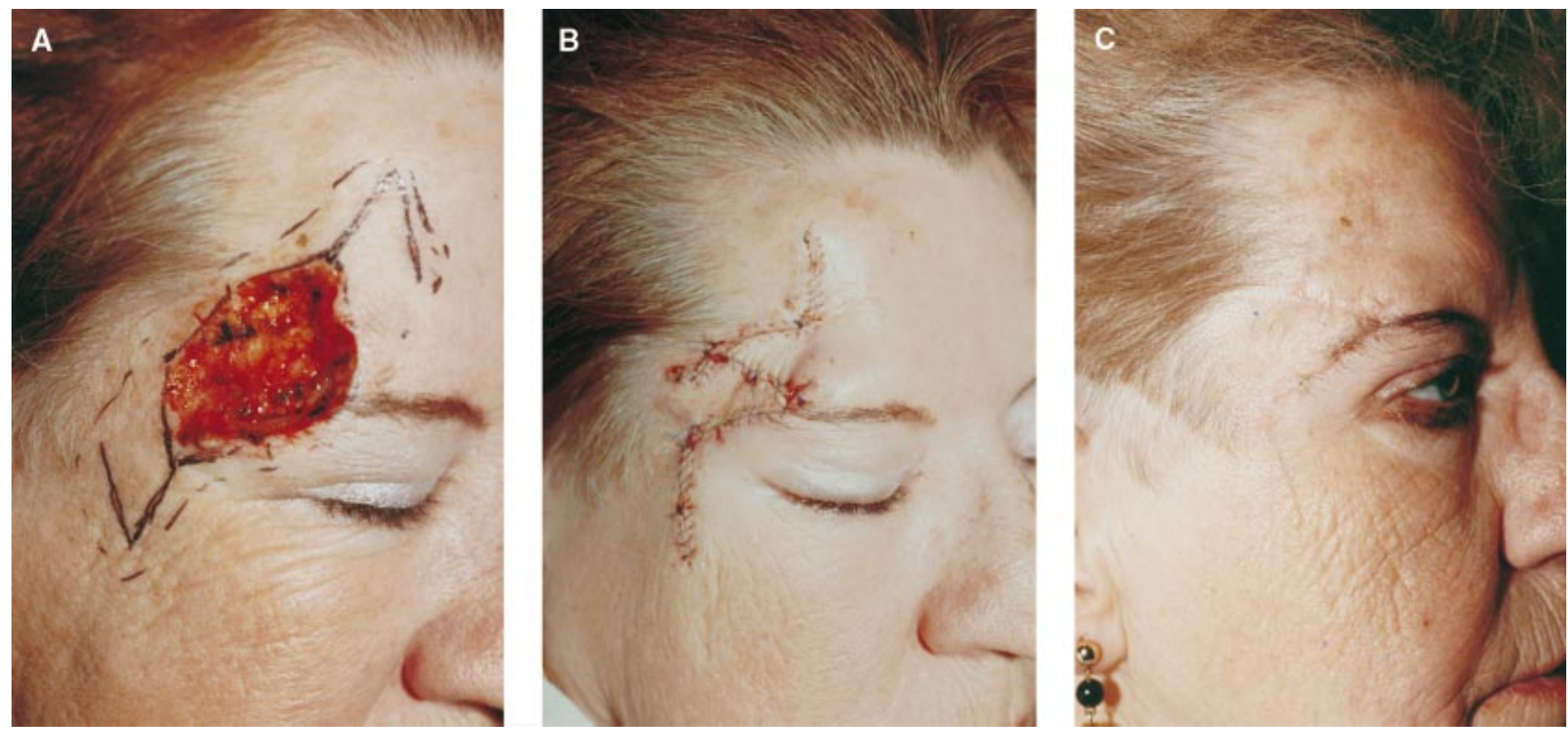

Figure 6. Patient 6. A 61-year-old woman with a significant smoking history had a $3.0 \mathrm{~cm} \times 4.0 \mathrm{~cm}$ defect at the forehead-eyebrow-temple area extending to the muscle following Mohs excision of a BCC. Loose adjacent tissue was identified on the forehead and temple. A) Two rhomboid shaped flaps were designed adjacent to the defect, each responsible for closing half of the defect. Tension vectors resulted in minimal distortion of the free margin at the eyebrow-eyelid. B) Scars lie primarily in rhitides and natural skin lines (6B). However, the lateral portion of the scar is perpendicular to the natural eyelid-cheek lines. A Z-plasty was offered to correct this perpendicular line, but the patient declined revisional surgery and was satisfied with the result. The temporal branch of the facial nerve and temporal artery are located within the area of reconstruction. C) Follow-up at 1 month. Massage and time will improve the final cosmetic appearance. 
fect. Transposition, hinge, and staged interpolated (pedicle) flaps move tissue in a lifting fashion. A comprehensive discussion of flap design has been performed previously by several authors. ${ }^{1-5}$

Prior to deciding which flap to use for a given defect, a mental assessment of three known results from flap transfer should be considered. First, where are the tension vectors that cause primary and secondary motion from transferring the flap? Do these vectors respect anatomic free margins? Second, where are the final incision lines? Do they respect cosmetic units, boundaries, and relaxed free skin tension lines? And third, where is the redundant skin, standing cutaneous deformity, or standing cone that results from tissue transfer? For defects that involve a large portion of a cosmetic unit, removal of the remaining portion of the unit will result in scars lying in cosmetic unit boundary lines and enhance the final cosmetic result.

Assessment of two main skin properties that enable tissue movement should also be evaluated during planning of a skin flap: elasticity and mobility. Elasticity is based on the skin's ability to stretch and is variable according to anatomic site. For example, cheek skin is more elastic than forehead skin. Mobility is based on the ability of skin to move from one point to another. The degree of mobility is often determined by the tissue underlying the skin, such as the underlying galea aponeurotica, muscle, and superficial aponeurotic system (SMAS). A thorough understanding of deeper anatomy enables the surgeon to determine the optimal plane of dissection. For example, scalp flaps move better when incisions are through the underlying galea, while cutaneous flaps on the nose and lip often move better with incisions through the underlying SMAS. A complete understanding of specific regional anatomy is required to prevent damage to underlying structures such as nerves, vessels, cartilaginous framework, and more complex structures such as the nasal valve.

\section{Case Examples}

Using a series of six similar defects (Figures 1-6) located in the forehead-temple-eyebrow area, all repaired with a different flap, the concept of tissue movement is demonstrated. First, the area of loose adjacent tissue is identified by pinching, pulling, and pushing adjacent tissue. The area of greatest redundancy of skin is usually the preferred location for harvesting a flap. The specific flap planned is then de- signed based on the three events previously discussed. Use of a regional "cookbook" approach is limiting, impedes thinking and creativity, and may not result in the best cosmetic and functional outcome. In addition, patient considerations should be assessed in each case. With this approach, multiple different flap designs can often be utilized for a given defect with similar results. In addition, scar revision ranging from Z-plasty to other incisional techniques and dermabrasion or laser abrasion can be utilized to achieve optimal cosmesis. These six case examples demonstrate use of the concept of sliding and lifting tissue movement with six different flaps utilized to repair six similar defects (Figures 1-6).

\section{The Fundamental Oncologic Principle}

The fundamental oncologic principle is tumor clearance first, reconstruction second. Prior to any flap reconstruction, comprehension of the fundamental oncologic principle is paramount. The best-designed flap reconstruction is a failure if performed without complete tumor extirpation. Two separate events should occur with surgical removal of a tumor. First and foremost is complete excision, which can be obtained with permanent section control, standard frozen-section control, or Mohs surgery. The best method is primarily dependent on the ability and experience of the surgeon and pathologist, and the basic tumor factors relative to the risk of subclinical invasion and recurrence. ${ }^{7}$ The second event involves assessment of reconstructive options. An interdisciplinary approach is useful in cases where another reconstructive surgeon will perform a better reconstruction.

\section{References}

1. Summers BK, Siegle RJ. Facial cutaneous reconstructive surgery: general aesthetic principles. J Am Acad Dermatol 1993;29:669-81.

2. Summers BK, Siegle RJ. Facial cutaneous reconstructive surgery: facial flaps. J Am Acad Dermatol 1993;29:917-41.

3. Burget GC, Menick FJ, eds. Aesthetic reconstruction of the nose. St. Louis: Mosby, 1994.

4. Baker SR, Swanson NA, eds. Local flaps in facial reconstruction. St. Louis: Mosby, 1995.

5. Salasche SJ, Bernstein G, Senkarik M, Davis K, eds. Surgical anatomy of the skin. East Norwalk, CT: Appleton \& Lange, 1988.

6. Swanson NA. Classifications, definitions, and concepts in flap surgery. In: Cummings CW, ed. Local flaps in facial reconstruction. St. Louis: Mosby, 1995:63-74.

7. Swanson NA, Johnson TM. Management of basal and squamous cell carcinoma. In: Cummings CW, ed. Otolaryngology head and neck surgery, 3rd ed. St. Louis: Mosby, 1998:486-501. 